\title{
JP-CoMP with Antenna Selection for Multi-Carrier C-RAN with Finite Backhaul Capacity
}

\author{
Ganesh Venkatraman, Antti Tölli, Jarkko Kaleva, Markku Juntti \\ Centre for Wireless Communications (CWC) - Radio Technologies, \\ University of Oulu, Finland - FI-90014, P.O. Box 4500, Email: firstname.lastname@oulu.fi
}

\begin{abstract}
We consider a multi-carrier joint processing (JP) coordinated multi-point (CoMP) cellular system wherein the beamformers are designed by a centralized controller (CC). Those are subsequently fed back to the respective base stations (BSs) via finite capacity backhaul link. Hence, we design transmit beamformers at the $\mathrm{CC}$ that associate users to BSs depending on their backhaul capacity and users channel state to address this problem. Upon identifying the active links, user multiplexing and scheduling over spatial and frequency dimensions are performed in conjunction with the beamformer design for all BSs subject to the objective of minimizing the number of backlogged packets at the $\mathrm{CC}$. Since the beamformers are notified to the respective BSs along with the data, the backhaul utilization must include the overhead incurred by the quantized beamformers, which has higher precision as it is used by all data symbols transmitted over a coherence block. In this paper, we assume fixed quantization levels for each complex entry in the beamformers. Therefore, we select a subset of entries for each beamformer to reduce the overhead incurred by the active antennas, which when removed has the minimal impact on the user rate. With the above mentioned constraints and requirements, the beamformer design becomes a nonconvex combinatorial problem. Therefore, we provide a solution for the proposed formulation by employing successive convex approximation technique to handle the nonconvexity and the combinatorial search involving binary variables are replaced by a linear bounds along with a sparsity inducing term in the objective. The proposed method is useful only when the backhaul usage due to the beamformers is significantly larger when compared to the overhead incurred by the active users data. Numerical results are provided to illustrate the performance.
\end{abstract}

\section{INTRODUCTION}

We consider a cooperative multi-cell joint processing (JP) coordinated multi-point (CoMP) transmission wherein multiple base stations (BSs) coherently transmit the same data to users over an orthogonal frequency division multiplexing (OFDM) framework. Serving users by multiple BSs increases their throughput significantly [1]. However, due to finite backhaul capacity, it is often not possible to include all BSs in the network for CoMP transmission as it requires the users' data to be conveyed through the backhaul. Therefore, due to the limitations imposed by the finite backhaul capacity, the number of cooperating BSs is restricted to a subset of BSs only. In such cases, the beamformer design becomes more challenging as it involves both user association and beamformer design for the chosen subset of users [2]-[6].

Typically, downlink transmissions are focused to provide certain quality-of-service (QoS) for users that are either directly or indirectly related to the minimization of queued packets with certain priorities imposed by the physical layer.
Therefore, we focus on the queue minimization objective for the design of beamformers at the centralized controller (CC). Earlier studies on queue minimization were summarized in the survey paper [7]. In particular, the problem of power allocation to minimize the number of backlogged packets was considered in [8] for a single-input single-output (SISO) OFDM system. Since the beamformers are designed by the $\mathrm{CC}$, both users' data and beamformers are signaled via backhaul to the respective $\mathrm{BSs}$ by the $\mathrm{CC}$. Therefore, to reduce the backhaul utilization, the beamformers are quantized with enough number of bits to guarantee sufficient accuracy for a JP CoMP transmission. The problem of multi-antenna beamformer quantization was studied extensively in [9]-[13].

Due to channel coherency in both time and frequency dimensions, multiple data symbols in a frame are precoded with the same beamformer. Therefore, it is efficient to send beamformers and data symbols from finite alphabets separately to the respective BSs. However, if the BSs act as a remote radio head, then signaling the beamformers and data symbols separately is not an efficient choice [5], [6]. Thus, as an extension to [3], we consider the signaling of both users' data and the beamformers from the $\mathrm{CC}$ to all the BSs, since both are available only at the $\mathrm{CC}$. As the number of antennas increases, the overhead incurred by the quantized beamformers will be significant enough to limit either the number of served users or the rate. Thus, we consider antenna selection as a candidate to reduce the beamformer overhead.

\section{System Model \& Problem Formulation}

We consider a model with $N_{B}$ BSs, each equipped with $N_{T}$ transmit antennas, serving $K$ single antenna users over $N$ OFDM sub-channels or coherence bands. ${ }^{1}$ The set of all BS in the system is denoted by $\mathcal{B}=\left\{1,2, \ldots, N_{B}\right\}$ and the set of all users is represented by $\mathcal{U}=\{1,2, \ldots, K\}$. The received symbol $y_{k, n}$ of user $k$ on the $n$th sub-channel is given by

$$
y_{k, n}=\sum_{b \in \mathcal{B}} \sum_{k \in \mathcal{U}} \mathbf{h}_{b, k, n} \mathbf{m}_{b, k, n} d_{k, n}+e_{k, n}
$$

where $\mathbf{h}_{b, k, n} \in \mathbb{C}^{1 \times N_{T}}$ denotes the channel between user $k$ and BS $b$ on the $n$th OFDM sub-channel. The transmit data symbol $d_{k, n}$ corresponding to each user $k$ has $\mathbb{E}\left[\left|d_{k, n}\right|^{2}\right]=1$ and $e_{k, n}$ is a sample of circularly symmetric additive white

\footnotetext{
${ }^{1}$ The term sub-channel refers to a group of frequency resources for which the channel is assumed to be constant. Hence, the beamformers are designed for a group of sub-carriers over which multiple data symbols are transmitted.
} 
complex Gaussian noise drawn from $\mathcal{C N}\left(0, N_{0}\right)$. The transmit beamformers $\mathbf{m}_{b, k, n} \in \mathbb{C}^{N_{T} \times 1}$, used to transmit data to user $k$, are designed by the $\mathrm{CC}$ and provided to the respective BSs in $\mathcal{B}$ for coordinated transmission. The received signalto-interference-plus-noise ratio (SINR) of user $k$ is given as

$$
\gamma_{k, n}=\frac{\left|\sum_{b \in \mathcal{B}} \mathbf{h}_{b, k, n} \mathbf{m}_{b, k, n}\right|^{2}}{\sum_{k^{\prime} \in \mathcal{U} \backslash k}\left|\sum_{b \in \mathcal{B}} \mathbf{h}_{b, k, n} \mathbf{m}_{b, k^{\prime}, n}\right|^{2}+N_{0}}
$$

by assuming that the users are served by all BSs coherently.

Let $Q_{k}$ be the number of backlogged packets destined for user $k$ at a given scheduling instant. The queue dynamics of user $k$ are modeled using the Poisson arrival process with the average number of packet arrivals of $\Lambda_{k}=\mathbf{E}_{i}\left\{\lambda_{k}\right\}$ packets or bits, where $\lambda_{k}[i] \sim \operatorname{Pois}\left(\Lambda_{k}\right)$ represents the instantaneous number of packets arriving for user $k$ at the $i$ th time instant. The total number of queued packets at the $(i+1)$ th instant for user $k$, denoted as $Q_{k}[i+1]$, is given by

$$
Q_{k}[i+1]=\max \left\{Q_{k}[i]-t_{k}[i], 0\right\}+\lambda_{k}[i]
$$

where $t_{k}$ denotes the number of transmitted packets or bits for user $k$ over $N$ OFDM sub-channels. The maximum transmission rate achieved in the $i$ th slot is given by $t_{k}[i] \leq$ $\sum_{n=1}^{N} \log _{2}\left(1+\gamma_{k, n}[i]\right)$ for a SINR $\gamma_{k, n}$. Note that $t_{k}$ and $Q_{k}$ are represented by the same units, i.e., in bits defined per channel use. We drop the time index $i$ for clarity reasons.

To minimize the number of backlogged packets associated with the users, we consider CoMP model with the objective

$$
\underset{t_{k, n}}{\operatorname{minimize}} \sum_{k \in \mathcal{U}} a_{k}\left|Q_{k}-\sum_{n=1}^{N} t_{k, n}\right|_{q}
$$

where $t_{k, n}$ is the rate seen by user $k$ on the $n$th sub-channel and $a_{k}$ is a constant which can be used to emphasize the user priority. The exponent $q \in \mathbb{Z}_{++}$determines how the backlogged packets of the users should be treated [14].

We assume that the channel state information (CSI) is available at the $\mathrm{CC}$ for designing transmit beamformers as it involves BSs to CC traffic. Even though the CSI has a finite resolution, it can be improved by differential feedback due to time-correlated nature of the channels. However, due to the instantaneous arrival process and rapidly changing interference structure, transmit beamformers used by BSs need not be the same even if the associated channels are constant. Therefore, the backhaul link between the CC and BS is used to signal both data and the beamformers. Due to the finite backhaul capacity, transmit beamformers are quantized before signaling them to the corresponding BSs. We assume that each complex entry of the beamformer vector is quantized with $N_{Q}$ bits. Since multiple data symbols in a frame are precoded with a same beamformer, it is efficient to send beamformers and data symbols from finite alphabets separately to the respective BSs. However, if the BSs act as a remote radio head, then signaling the beamformers and data symbols separately is not an efficient choice [5]. Hence, we consider the former scenario.

Even if we assume that BS $b$ is serving user $k$, it cannot be ensured that user $k$ is served by BS $b$ on all sub-channels.
Hence, to evaluate the total utilization of the backhaul to serve user $k$ by BS $b$, we introduce a binary variable $c_{b, k, n} \in\{0,1\}$, which has one when user $k$ is served by BS $b$ on the $n$th subchannel and zero otherwise. Thus, the total signaling via the finite backhaul link between BS $b$ and the $\mathrm{CC}$ is given as

$$
\sum_{n=1}^{N} \sum_{k \in \mathcal{U}} c_{b, k, n} t_{k, n}+N_{Q} N_{T} \sum_{n=1}^{N} \sum_{k \in \mathcal{U}} c_{b, k, n} \leq B_{b}
$$

where $N_{Q} N_{T}$ is the total number of bits required to signal the beamformer and $B_{b}$ is the respective backhaul link capacity.

Now, the optimization variable $t_{k, n}$ in (4) is determined by the SINR $\gamma_{k, n}$, which in turn depends on the transmit beamformers by (2). To ensure that $c_{b, k, n}$ determines whether BS $b$ is serving user $k$ over a sub-channel $n$ or not, the binary variable $c_{b, k, n}$ should be associated with beamformer as

$$
\left\|\mathbf{m}_{b, k, n}\right\|^{2} \leq c_{b, k, n} \nu_{b, k, n}
$$

where $\nu_{b, k, n}$ is an additional optimization variable, which corresponds to the power allocated by BS $b$ to user $k$ on sub-channel $n$. Therefore, if either $c_{b, k, n}$ or $\nu_{b, k, n}$ is zero, the respective beamformer $\mathbf{m}_{b, k, n}$ will be a zero vector, thereby ensuring the absence of transmission from BS $b$ to user $k$ on the $n$th sub-channel. Let $\mathcal{O}=\{0,1, \ldots, N\}$ be the set of all sub-channel indices and $\{\mathbf{m}\} \triangleq\left\{\mathbf{m}_{b, k, n}\right\}$, $\forall b \in \mathcal{B}, \forall k \in \mathcal{U}, \forall n \in \mathcal{O}$ be the collection of all transmit beamformers. Similarly, $\{\mathbf{t}\}$ and $\{\boldsymbol{\nu}\}$ denotes the collection of all users rates from each sub-channel and the transmit power from all BSs to each user. Now, the problem of designing transmit beamformers is given as

$$
\begin{array}{cl}
\underset{\begin{array}{c}
\{\mathbf{t}\},\{\mathbf{m}\}, \\
\{\gamma\},\{\boldsymbol{\nu}\},\{\mathbf{c}\}
\end{array}}{\operatorname{minimize}} & \sum_{k \in \mathcal{U}} a_{k}\left|Q_{k}-\sum_{n=1}^{N} t_{k, n}\right|_{q} \\
\text { subject to } & \log \left(1+\gamma_{k, n}\right) \geq t_{k, n} \\
& \sum_{k \in \mathcal{U}} \sum_{n=1}^{N} \nu_{b, k, n} \leq P_{\max }, \forall b \\
& (5),(6), \text { and } c_{b, k, n} \in\{0,1\}, \forall b, \forall k, \forall n
\end{array}
$$

where $\gamma_{k, n}$ is defined in (2). The constraint (7c) is included to limit the total transmit power from each BS within $P_{\max }$. The constraint (7b) introduces $t_{k, n}$ as an under-estimator for the rate seen by user $k$ on sub-channel $n$, since $t_{k, n}=\log (1+$ $\gamma_{k, n}$ ) cannot be handled directly in (7) as a convex constraint.

\section{BEAMFORMER DESIGN \& QUANTIZATION OVERHEAD}

To solve (7), we relax the SINR expression in (2) as

$$
\begin{aligned}
\frac{\left|\sum_{b \in \mathcal{B}} \mathbf{h}_{b, k, n} \mathbf{m}_{b, k, n}\right|^{2}}{b_{k, n}} & \geq \gamma_{k, n} \\
\sum_{k^{\prime} \in \mathcal{U} \backslash k}\left|\sum_{b \in \mathcal{B}} \mathbf{h}_{b, k, n} \mathbf{m}_{b, k^{\prime}, n}\right|^{2}+N_{0} & \leq b_{k, n}
\end{aligned}
$$

since the expression (2) cannot be handled directly. We assume that the beamformer precision is high enough to guarantee sufficient accuracy. Eq. (8a) is an under-estimator for $\gamma_{k, n}$ and $b_{k, n}$ in (8b) is a over-estimator for the total interference 
seen by user $k$ on sub-channel $n$. Even after relaxing (2) with (8), the problem is not convex due to (8a) and (5). Hence, we adopt successive convex approximation (SCA) wherein each nonconvex set is replaced by a convex subset, which can be solved iteratively until convergence [14], [15].

Let us begin by considering an equivalent representation for the SINR expression in (8a) for user $k$ on sub-channel $n$ as

$$
\gamma_{k, n} \leq\left(b_{k, n}{ }^{-1}\left|\overline{\mathbf{h}}_{k, n} \overline{\mathbf{m}}_{k, n}\right|^{2}\right.
$$

where the stacked channel vector $\overline{\mathbf{h}}_{k, n} \in \mathbb{C}^{1 \times|\mathcal{B}| N_{T}}$ and transmit beamformer $\overline{\mathbf{m}}_{k, n} \in \mathbb{C}^{|\mathcal{B}| N_{T} \times 1}$ are defined as

$$
\begin{aligned}
\overline{\mathbf{h}}_{k, n} & \triangleq\left[\mathbf{h}_{\mathcal{B}(1), k, n}, \mathbf{h}_{\mathcal{B}(2), k, n}, \ldots, \mathbf{h}_{\mathcal{B}(|\mathcal{B}|), k, n}\right. \\
\overline{\mathbf{m}}_{k, n} & \triangleq\left[\mathbf{m}_{\mathcal{B}(1), k, n}^{\mathrm{T}}, \mathbf{m}_{\mathcal{B}(2), k, n}^{\mathrm{T}}, \ldots, \mathbf{m}_{\mathcal{B}(|\mathcal{B}|), k, n}^{\mathrm{T}} \mathrm{T}\right.
\end{aligned}
$$

The fractional term in (9) is of quadratic-over-linear form, and can be bounded by the linear first order Taylor series as

$$
\begin{aligned}
& \frac{\left|\overline{\mathbf{h}}_{k, n} \overline{\mathbf{m}}_{k, n}\right|^{2}}{b_{k, n}} \geq \mathcal{L}_{k, n}^{(i)}\left(\overline{\mathbf{m}}_{k, n}, b_{k, n} ; \overline{\mathbf{m}}_{k, n}^{(i)}, b_{k, n}^{(i)} \triangleq\right. \\
&\left.2 \frac{\overline{\mathbf{m}}_{k, n}^{(i)} \overline{\mathbf{h}}_{k, n}^{\mathrm{H}} \overline{\mathbf{h}}_{k, n}}{b_{k, n}^{(i)}} \overline{\mathbf{m}}_{k, n}-\overline{\mathbf{m}}_{k, n}^{(i)}\right) \\
& \quad+\frac{\left|\overline{\mathbf{h}}_{k, n} \overline{\mathbf{m}}_{k, n}^{(i)}\right|^{2}}{b_{k, n}^{(i)}}\left(1-\frac{b_{k, n}-b_{k, n}^{(i)}}{b_{k, n}^{(i)}}\right)
\end{aligned}
$$

where $\mathcal{L}_{k, n}^{(i)}\left(\overline{\mathbf{m}}_{k, n}, b_{k, n}\right)$ is a linear under-estimator for the r.h.s term in (9) and $\left\{\overline{\mathbf{m}}_{k, n}^{(i)}, \overline{\mathbf{b}}_{k, n}^{(i)}\right\}$ are the operating points that are updated in each step by the solution obtained from the previous SCA step. Due to the quasi-concavity of $c_{b, k, n} t_{k, n}$, the backhaul constraint (5) becomes a nonconvex one. Therefore, to find a convex approximation for (5), we express $c_{b, k, n} t_{k, n}$ by an equivalent difference-of-convex (DC) function as

$$
c_{b, k, n} t_{k, n}=\frac{1}{4}\left[\left(c_{b, k, n}+t_{k, n}\right)^{2}-\left(c_{b, k, n}-t_{k, n}\right)^{2} .\right.
$$

In spite of using (12), (5) is still nonconvex due to the concave nature of the second term in (12). Hence, we linearize it as

$$
\begin{aligned}
& \mathcal{F}_{b}^{(i)}\left(c_{b, k, n}, t_{k, n} ; c_{b, k, n}^{(i)}, t_{k, n}^{(i)} \triangleq \frac{1}{4} \sum_{n=1}^{N} \sum_{k \in \mathcal{U}}\left[\left(c_{b, k, n}+t_{k, n}\right)^{2}\right.\right. \\
+ & \left.\left.c_{b, k, n}^{(i)}-t_{k, n}^{(i)}\right)^{2}-2 \quad c_{b, k, n}^{(i)}-t_{k, n}^{(i)}\right)\left(c_{b, k, n}-t_{k, n}\right)
\end{aligned}
$$

where the operating points $c_{b, k, n}^{(i)}$ and $t_{k, n}^{(i)}$ are the solutions for $c_{b, k, n}$ and $t_{k, n}$, respectively, from the $(i-1)$ th SCA iteration.

Now, by replacing (8a) with (11) and (5) by (13), we obtain a mixed-integer quadratic programming (MIQP) subproblem for each SCA iteraiton $i$ as

$$
\begin{array}{ll}
\underset{\begin{array}{c}
\{\mathbf{t}\},\{\mathbf{m}\},\{\mathbf{b}\}, \\
\{\boldsymbol{\gamma}\},\{\boldsymbol{\nu}\},\{\mathbf{c}\}
\end{array}}{\operatorname{minimize}} & \sum_{k \in \mathcal{U}} a_{k}\left|Q_{k}-\sum_{n=1}^{N} t_{k, n}\right|_{q} \\
\text { subject to } & \mathcal{L}_{k, n}^{(i)}\left(\overline{\mathbf{m}}_{k, n}, b_{k, n} ; \overline{\mathbf{m}}_{k, n}^{(i)}, b_{k, n}^{(i)}\right) \geq \gamma_{k, n} \\
& \mathcal{F}_{b}^{(i)}\left(c_{b, k, n}, t_{k, n} ; c_{b, k, n}^{(i)}, t_{k, n}^{(i)}\right.
\end{array}
$$

$$
\begin{aligned}
& +\mu N_{Q} N_{T} \sum_{n=1}^{N} \sum_{k \in \mathcal{U}} c_{b, k, n} \leq B_{b}, \forall b \\
& \text { (7b) - (7d) and (8b) }
\end{aligned}
$$

where $\gamma_{k, n}$ is positive even after relaxation, since a negative value for $\gamma_{k, n}$ reduces $t_{k, n}$ in (7b) for a non-zero beamformer entry $\mathbf{m}_{b, k, n}$, thereby consuming power without minimizing the objective. Typically, the users' data symbols that are mapped to a coherence block for transmission, are precoded by a common beamformer. Therefore, the traffic caused by the quantized beamformers is only a fraction of the respective data symbols, which is captured by the constant $\mu$ in (14c).

Due to the complexity involved with (14), we propose a relaxation for the binary decision variable as $c_{b, k, n} \in[0,1]$. However, by doing so, the resulting solution for $c_{b, k, n}$ may not be binary. Therefore, to encourage binary solution for the linear variable $c_{b, k, n}$, we include an additional constraint in the formulation to enforce sparsity in the solution. One such function is the entropy measure, defined as

$$
\mathcal{H}_{b}\left(\mathbf{c}_{b}\right) \triangleq-\sum_{k=1}^{K} \sum_{n=1}^{N} c_{b, k, n} \log \left(c_{b, k, n}\right)
$$

where $\mathbf{c}_{b}$ is a vector formed by stacking all $c_{b, k, n}, \forall k$ and $\forall n$ corresponding to $\mathrm{BS} b$. In order to ensure binary outcome for $\mathbf{c}_{b}, \mathcal{H}_{b}\left(\mathbf{c}_{b}\right)$ in (15) must be equal to or less than zero. By doing so, the constraint with $\mathcal{H}_{b}\left(\mathbf{c}_{b}\right) \leq 0$ will become nonconvex due to the concavity of the entropy function. Thus, we relax the constraint by the partial Lagrangian approach with dual variables $\tau_{b}, \forall b \in \mathcal{B}$ as

$$
\begin{array}{cl}
\underset{\begin{array}{c}
\{\mathbf{t}\},\{\mathbf{m}\},\{\mathbf{b}\} \\
\{\boldsymbol{\gamma}\},\{\boldsymbol{\nu}\},\{\mathbf{c}\}
\end{array}}{\operatorname{minimize}} & \sum_{k \in \mathcal{U}} a_{k}\left|Q_{k}-\sum_{n=1}^{N} t_{k, n}\right|_{q}+\sum_{b \in \mathcal{B}} \tau_{b} \mathcal{H}_{b}\left(\mathbf{c}_{b}\right) \\
\text { subject to } & 0 \leq c_{b, k, n} \leq 1, \forall b, \forall k, \forall n \\
& (7 \mathbf{b})-(7 \mathrm{c}),(8 \mathrm{~b}),(14 \mathrm{~b}), \text { and }(14 \mathrm{c})
\end{array}
$$

which is similar to reweighted $\ell_{1}$ minimization method in [16].

The problem in (16) is nonconvex due to the concave nature of the entropy function. Therefore, to formulate a tractable problem, we employ the SCA technique to find a linear overestimator for the entropy function as

$$
\begin{gathered}
\left.\mathcal{H}_{b}\left(\mathbf{c}_{b}\right) \leq \mathcal{H}_{b}^{(i)}\left(\mathbf{c}_{b} ; \mathbf{c}_{b}^{(i)}\right) \triangleq-\sum_{k=1}^{K} \sum_{n=1}^{N} c_{b, k, n}^{(i)} \log c_{b, k, n}^{(i)}\right)- \\
\left.\sum_{k=1}^{K} \sum_{n=1}^{N}\left(1+\log c_{b, k, n}^{(i)}\right)\right)\left(c_{b, k, n}-c_{b, k, n}^{(i)}\right)
\end{gathered}
$$

which is a first order Taylor approximation of the entropy function. Now, for fixed $\tau_{b}, \forall b$, the relaxed convex subproblem for the SCA iteration $i$ is given as

$$
\begin{aligned}
\underset{\substack{\{\mathbf{t}\},\{\mathbf{m}\},\{\mathbf{b}\}\},\{\boldsymbol{\gamma}\},\{\boldsymbol{\nu}\},\{\mathbf{c}\}}}{\operatorname{minimize}} \sum_{k \in \mathcal{U}} a_{k} \mid Q_{k} & -\left.\sum_{n=1}^{N} t_{k, n}\right|_{q} \\
& +\sum_{b \in \mathcal{B}} \tau_{b}^{(i)} \mathcal{H}_{b}^{(i)}\left(\mathbf{c}_{b} ; \mathbf{c}_{b}^{(i)}\right)
\end{aligned}
$$


subject to $\quad 0 \leq c_{b, k, n} \leq 1, \forall b, \forall k, \forall n$

$$
(7 b)-(7 c),(8 b),(14 b), \text { and }(14 c)
$$

where $\tau_{b}^{(i)}, \forall b$ are the fixed dual variables, given by the subgradient update as

$$
\tau_{b}^{(i+1)}=\tau_{b}^{(i)}+\rho^{(i)}\left[\mathcal{H}_{b}^{(i)}\left(\mathbf{c}_{b}^{(i+1)} ; \mathbf{c}_{b}^{(i)}\right)^{+}\right.
$$

where $\mathbf{c}_{b}^{(i+1)}$ is the solution for $\mathbf{c}_{b}$ in the $i$ th iteration, $\rho$ is a fixed step size with $\rho^{(i)}>0$ to ensure sparse solution upon convergence and $[x]^{+} \triangleq \max (x, 0)$. As $c_{b, k, n} \rightarrow\{0,1\}$, $\mathcal{H}_{b}\left(\mathbf{c}_{b}\right) \rightarrow 0$, and thus $\mathcal{H}_{b}^{(i)}\left(\mathbf{c}_{b} ; \mathbf{c}_{b}^{(i)}\right)$ has no effect on (18a).

\section{BeAmformer Design with Antenna Selection}

Due to the fixed quantization levels for all complex entries in the beamformers $\mathbf{m}_{b, k, n}$, the backhaul overhead increases linearly by $\mu N_{Q}$ as the number of antennas in each BSs scales-up. Therefore, to reduce the beamformer overhead in the backhaul, we can adopt either one of the following approaches.

- Instead of using a fixed high precision quantization levels, the beamformers corresponding to each cooperating BSs can be quantized with different resolutions based on their respective contribution in the users' rates. However, the problem formulation will be difficult as it involves both beamformers and their respective quantization levels as an optimization variables.

- Alternatively, by keeping the quantization levels fixed and sufficiently high enough for all beamformers to ignore distortion, the number of active transmit elements can be reduced by setting zero power to insignificant antennas in the beamformers, thereby utilizing the backhaul efficiently to increase the users data rate.

The first approach is studied in [17], whereas this work is focused on the approach of reducing the number of active antennas used by the BSs to serve users in a CoMP transmission.

Now, to identify a subset of active antennas for each transmission link, we introduce an additional binary variable for each antenna to identify the respective status as

$$
\left|m_{b, k, n}(l)\right|^{2} \leq a_{b, k, n}(l) w_{b, k, n}(l)
$$

where $a_{b, k, n}(l) \in\{0,1\}$ and $\mathbf{w}_{b, k, n} \in \mathbb{R}_{+}^{N_{T} \times 1}$ denote the status and the power of the $l$ th complex entry in $\mathbf{m}_{b, k, n}$, respectively. Now, by using the newly introduced variable $\mathbf{w}_{b, k, n}$, the constraint (6) can be expressed as

$$
\left\|\mathbf{m}_{b, k, n}\right\|^{2} \leq c_{b, k, n}\left(\sum_{l=1}^{N_{T}} w_{b, k, n}(l)\right)
$$

and the total power budget (7c) can also be rewritten as

$$
\sum_{k \in \mathcal{U}} \sum_{n=1}^{N} \sum_{l=1}^{N_{T}} w_{b, k, n}(l) \leq P_{\max }, \forall b
$$

thereby eliminating the need for $\nu_{b, k, n}$ in the problem.

Now, by introducing antenna selection in the problem formulation, the backhaul can be utilized efficiently by finding a trade-off between the amount of bits allocated for users' data and for beamformer notifications. In order to do so, the BS specific backhaul utilization (5) should be modified to reflect the savings due to antenna selection as $\forall b \in \mathcal{B}$

$$
\sum_{n=1}^{N} \sum_{k \in \mathcal{U}}\left[c_{b, k, n} t_{k, n}+\mu N_{Q} c_{b, k, n} \sum_{l=1}^{N_{T}} a_{b, k, n}(l) \leq B_{b},\right.
$$

where $\mu$ is the ratio between beamformer and the number of data symbols for which it is applied. Furthermore, we should include an additional overhead to notify the beamformer entries that are not present in the feedback from $\mathrm{CC}$ to the respective BSs for each active transmission.

The notification overhead is given by the number of bits required to notify $\mathbf{a}_{b, k, n}$ for each active link. The overhead can be reduced if the $\mathrm{CC}$ signals only the missing beamformer entries after notifying the active complex values with $N_{Q}$ bits each. Since the number of disabled antennas is usually small as it reduces the achievable throughput, this would be an ideal choice as it requires $\log _{2}\left(N_{T}\right)$ bits for each disabled index. Therefore, if the number of inactive antenna entries is small when compared to $N_{T}$, then the overhead will be given by

$$
\left.\zeta_{b, k, n} \triangleq \log _{2}\left(N_{T}\right) \quad N_{T}-\sum_{l=1}^{N_{T}} a_{b, k, n}(l)\right) .
$$

However, if the number of disabled entries increases, $\zeta_{b, k, n} \gg$ $N_{T}$, then sending the whole vector $\mathbf{a}_{b, k, n}$ will be an efficient choice as it requires only $N_{T}$ bits. Therefore, we have to choose between the two signaling techniques, i.e.,

- to signal only the disabled entries after the transmission of active complex values with $N_{Q}$ bits each or

- to signal the whole vector $\mathbf{a}_{b, k, n}$ when $\zeta_{b, k, n} \geq N_{T}$.

Additionally, for the following two cases, there is no need for any signaling, namely, (i) when a user is not served by a BS on a sub-channel, i.e., $c_{b, k, n}=0$, and (ii) if all the antennas are active for a transmission by BS to user on a sub-channel.

The above mentioned requirements can be implemented by the following notification constraint as

$$
\left.\min \left\{N_{T}, \log _{2}\left(N_{T}\right) \quad N_{T}-\sum_{l=1}^{N_{T}} a_{b, k, n}(l)\right)\right\}
$$

If the notification type is based on overhead minimization, then the signaling of either the disabled entries or the whole binary vector can be identified implicitly by opting for whole $\mathbf{a}_{b, k, n}$ when $N_{T} \leq \zeta_{b, k, n}$, thereby avoiding an additional bit. Hence, by including (25) in (23), we get the backhaul utilization as

$$
\begin{aligned}
B_{b} & \geq \sum_{n=1}^{N} \sum_{k \in \mathcal{U}}\left[\mu N_{Q} c_{b, k, n} \sum_{l=1}^{N_{T}} a_{b, k, n}(l)\right)+c_{b, k, n} t_{k, n} \\
& \left.\left.+\mu \min \left\{N_{T}, \log _{2}\left(N_{T}\right) \quad N_{T}-\sum_{l=1}^{N_{T}} a_{b, k, n}(l)\right)\right\}\right]
\end{aligned}
$$

Due to the presence of binary variables in (26), it is not possible to solve the beamformer design efficiently. Thus, we relax all the binary variables by a linear one as discussed in 
Section III along with a sparsity inducing entropy constraint $\mathcal{H}_{b}\left(\mathbf{a}_{b}\right) \leq 0$, where the function $\mathcal{H}_{b}$ is defined in (15).

Now, let us handle the terms in (26) separately. As earlier, the product term $c_{b, k, n} t_{k, n}$ cannot be handled directly due to quasi-concavity of the function. However, it can be represented by a DC form (12), and thus can be upper bounded by using (13). Then, we have quantized beamformer overhead, which is also a quasi-convex function due to the product of linear variables. Now, by using (12), the first term in (26) can be replaced by a suitable convex function similar to (13) as

$$
\begin{gathered}
\mathcal{F}_{b}^{(i)}\left(c_{b, k, n}, \mathbf{a}_{b, k, n} ; c_{b, k, n}^{(i)}, \mathbf{a}_{b, k, n}^{(i)} \triangleq\right. \\
\sum_{n=1}^{N} \sum_{k \in \mathcal{U}}\left[\left(c_{b, k, n}+\sum_{l=1}^{N_{T}} a_{b, k, n}(l)\right)^{2}+c_{b, k, n}^{(i)}-\sum_{l=1}^{N_{T}} a_{b, k, n}^{(i)}(l)\right)^{2} \\
\left.\left.-2 c_{b, k, n}^{(i)}-\sum_{l=1}^{N_{T}} a_{b, k, n}^{(i)}(l)\right)\left(c_{b, k, n}-\sum_{l=1}^{N_{T}} a_{b, k, n}(l)\right)\right] \times \frac{1}{4} \\
\geq \sum_{n=1}^{N} \sum_{k \in \mathcal{U}} c_{b, k, n} \sum_{l=1}^{N_{T}} a_{b, k, n}(l)
\end{gathered}
$$

where $\mathcal{F}_{b}^{(i)}\left(c_{b, k, n}, \mathbf{a}_{b, k, n} ; c_{b, k, n}^{(i)}, \mathbf{a}_{b, k, n}^{(i)}\right.$ is an approximate function for the $i$ th SCA iteration around $c_{b, k, n}^{(i)}$ and $\mathbf{a}_{b, k, n}^{(i)}$.

Finally, the notification overhead (25) is also nonconvex, and therefore it has to be replaced by a suitable convex upper bound to make (26) as a convex constraint in the joint queue minimization problem. Instead of finding an convex upper bound, we replace it by a linear interpolation between two values by introducing a new variable $\delta_{b, k, n}$ as

$$
\delta_{b, k, n} N_{T}+\left(1-\delta_{b, k, n}\right) \zeta_{b, k, n}
$$

and by restricting $0 \leq \delta_{b, k, n} \leq 1$. In order to show the equivalence between (28) and (25), let us analyze the behavior of (28). Since $\delta_{b, k, n}$ is a free variable, it can take any value between $[0,1]$ to reduce the overhead, which in turn minimizes the objective. Thus, if $\zeta_{b, k, n}<N_{T}$, then $\delta_{b, k, n}=0$ to reduce the notification overhead in the backhaul. On the contrary, if $\zeta_{b, k, n}>=N_{T}$, then $\delta_{b, k, n}=1$ for the same reason. However, when the link is inactive, i.e., $c_{b, k, n}=0$, then (28) will have non-zero values for all $0 \leq \delta_{b, k, n} \leq 1$ if $a_{b, k, n}=\mathbf{0}^{\mathrm{T}}$, and thus leading to a non-zero overhead. Nevertheless, by inspecting (26), we can see that this issue is avoided by having $c_{b, k, n}$ before the antenna status variable $\boldsymbol{a}_{b, k, n}$, since, when $c_{b, k, n}=0$, then $\mathbf{a}_{b, k, n}$ can take $\mathbf{1}^{\mathrm{T}}$, thereby leading to $\zeta_{b, k, n}=0$ and $\delta_{b, k, n}=0$ without contributing to the overhead. Similarly, if the link is active with all antennas enabled, i.e., $c_{b, k, n}=1$ and $a_{b, k, n}=\mathbf{1}^{\mathrm{T}}$, then $\delta_{b, k, n}$ will be zero to avoid the antenna status notification from the backhaul. Now, by using similar approximation as in (13) and (27) for the second term in (28), the total overhead is given by

$$
\begin{gathered}
\mu\left\{N _ { Q } \mathcal { F } _ { b } ^ { ( i ) } \left(c_{b, k, n}, \mathbf{a}_{b, k, n} ; c_{b, k, n}^{(i)}, \mathbf{a}_{b, k, n}^{(i)}+\delta_{b, k, n} N_{T}\right.\right. \\
+\sum_{n=1}^{N} \sum_{k \in \mathcal{U}}\left(\zeta_{b, k, n}-N_{T} \log _{2}\left(N_{T}\right) \delta_{b, k, n}\right.
\end{gathered}
$$

$$
\begin{array}{r}
+\log _{2}\left(N_{T}\right) \mathcal{F}_{b}^{(i)}\left(\delta_{b, k, n}, \mathbf{a}_{b, k, n} ; \delta_{b, k, n}^{(i)}, \mathbf{a}_{b, k, n}^{(i)}\right\} \\
+\mathcal{F}_{b}^{(i)}\left(c_{b, k, n}, t_{k, n} ; c_{b, k, n}^{(i)}, t_{k, n}^{(i)} \leq B_{b}\right.
\end{array}
$$

where $\delta_{b, k, n}^{(i)}$ is the fixed operating point of $\delta_{b, k, n}$ upon which the approximation is defined.

Alternatively, the backhaul constraint in (26) can also be expressed by using the linear interpolation variable $\delta_{b, k, n}$ as

$$
\begin{aligned}
& B_{b} \geq \sum_{n=1}^{N} \sum_{k \in \mathcal{U}} {\left[c_{b, k, n} t_{k, n}+\mu N_{Q} \sum_{l=1}^{N_{T}} a_{b, k, n}(l)+\right.} \\
&\left.\left.\delta_{b, k, n} c_{b, k, n} N_{T}+\left(1-\delta_{b, k, n}\right) \zeta_{b, k, n}\right)\right]
\end{aligned}
$$

where the difference between (26) and (30) is only from the usage of $c_{b, k, n}$. Again, the constraint (30) is nonconvex due to the functions on the r.h.s of the inequality. Thus, we resort to the SCA technique as earlier to handle the nonconvexity as in (13) and (27). The relaxed convex form of (30) is given as

$$
\begin{gathered}
\mu\left\{\sum_{n=1}^{N} \sum_{k \in \mathcal{U}} N_{Q} \sum_{l=1}^{N_{T}} a_{b, k, n}(l)+\sum_{n=1}^{N} \sum_{k \in \mathcal{U}}\left(\zeta_{b, k, n}\right.\right. \\
\left.-N_{T} \log _{2}\left(N_{T}\right) \delta_{b, k, n}\right)+\mathcal{F}_{b}^{(i)}\left(c_{b, k, n}, \delta_{b, k, n} ; c_{b, k, n}^{(i)}, \delta_{b, k, n}^{(i)}\right. \\
+\log _{2}\left(N_{T}\right) \mathcal{F}_{b}^{(i)}\left(\delta_{b, k, n}, \mathbf{a}_{b, k, n} ; \delta_{b, k, n}^{(i)}, \mathbf{a}_{b, k, n}^{(i)}\right\} \\
+\mathcal{F}_{b}^{(i)}\left(c_{b, k, n}, t_{k, n} ; c_{b, k, n}^{(i)}, t_{k, n}^{(i)} \leq B_{b} .\right.
\end{gathered}
$$

Using (31) in the problem has an impact on the variables $\mathbf{a}_{b, k, n}$ and $\delta_{b, k, n}$ compared to (29). In contrast to (29), if the link is inactive by setting $c_{b, k, n}=0$, then $\mathbf{a}_{b, k, n}=\mathbf{0}^{\mathrm{T}}$ and the variable $\delta_{b, k, n}=1$, thereby choosing $c_{b, k, n} N_{T}=0$ as the notification overhead. On the contrary, if all the links are active, then $\delta_{b, k, n}=0$ to choose $\zeta_{b, k, n}$ with zero value to avoid the notification overhead for this scenario as well. As a comparison between (29) and (31), we can see that due to the linear relaxation of $c_{b, k, n}, \delta_{b, k, n}$ and $\mathbf{a}_{b, k, n}$, the term $c_{b, k, n} \delta_{b, k, n}$ in (31) emphasize more on reducing the number of active antennas, since to disable a link and to minimize the backhaul overhead, $\sum_{l=1}^{N_{T}} a_{b, k, n}(l)$ should be minimized. Whereas in (29), $\mathbf{a}_{b, k, n} \rightarrow \mathbf{1}^{\mathrm{T}}$ when $c_{b, k, n} \rightarrow 0$ in order to avoid the notification overhead, and hence, it favors larger numbers of antennas for active transmission by finding a balance between the user rate and the beamformer overhead.

Now, by using the above approximations and constraints, an approximate queue minimizing beamformer design problem for the SCA iteration $i$ is given by

$$
\begin{array}{cc}
\underset{\begin{array}{c}
\{\mathbf{t}\},\{\mathbf{m}\},\{\mathbf{b}\} \\
\{\mathbf{w}\},\{\mathbf{c}\} \\
\{\boldsymbol{\gamma}\},\{\boldsymbol{\delta}\}\{\mathbf{a}\}
\end{array}}{\operatorname{minimize}} & \sum_{k \in \mathcal{U}} a_{k}\left|Q_{k}-\sum_{n=1}^{N} t_{k, n}\right|_{q}+\sum_{b \in \mathcal{B}} \tau_{b}^{(i)} \mathcal{H}_{b}^{(i)}\left(\mathbf{c}_{b} ; \mathbf{c}_{b}^{(i)}\right) \\
& +\sum_{b \in \mathcal{B}} \phi_{b}^{(i)} \mathcal{H}_{b}^{(i)}\left(\mathbf{a}_{b} ; \mathbf{a}_{b}^{(i)}\right) \\
\text { subject to } & \log \left(1+\gamma_{k, n}\right) \geq t_{k, n} \\
& \mathcal{L}_{k, n}^{(i)}\left(\overline{\mathbf{m}}_{k, n}, b_{k, n} ; \overline{\mathbf{m}}_{k, n}^{(i)}, b_{k, n}^{(i)} \geq \gamma_{k, n}\right.
\end{array}
$$




$$
\begin{aligned}
& 0 \leq c_{b, k, n} \leq 1,0 \leq \delta_{b, k, n} \leq 1 \\
& \mathbf{0}^{\mathrm{T}} \leq \mathbf{a}_{b, k, n} \leq \mathbf{1}^{\mathrm{T}}
\end{aligned}
$$

(8b), (20), (21), (22) and (29) or (31)

where the constants $\tau_{b}^{(i)}$ and $\phi_{b}^{(i)}$ are the dual variables corresponding to the entropy constraints of $c_{b, k, n}$ and $\mathbf{a}_{b, k, n}$, respectively. In each SCA iteration $i$, they are updated as

$$
\phi_{b}^{(i+1)}=\phi_{b}^{(i)}+\alpha^{(i)}\left[\mathcal{H}_{b}^{(i)}\left(\mathbf{a}_{b}^{(i+1)} ; \mathbf{a}_{b}^{(i)}\right)^{+}\right.
$$

where $\rho^{(i)}$ (defined in (19)) and $\alpha^{(i)}$ are the positive step sizes which can be an increasing sequence as $i \rightarrow \infty$, thereby ensuring sparsity upon convergence [16]. Once the solution is sparse, the entropy function will be zero and the goal becomes the original queue minimization objective defined in (4).

For initialization, the dual variables $\tau_{b}^{(0)}$ and $\phi_{b}^{(0)}$ are all set to zero so as to focus on the queue minimizing objective without emphasizing on the sparsity. As the SCA iteration increments, binary constraint is emphasized by the subgradient update in (19) and (33). The values of $\rho$ and $\alpha$ will be saturated upon reaching binary solution for the respective variables. If $\tau>\rho$, then (32) emphasizes more on active user links with highly random $\mathbf{a}_{b, k, n}$ having large entropy. However, as $i \rightarrow \infty, a_{b, k, n}(l) \rightarrow\{0,1\}$. Even though, we performed antenna selection for each link, i.e., the for each user, frequency and BS, it can be performed at the user level as well, i.e., by disabling a same set of antennas on all subchannels for a service between each BS and user. In this way,the notification overhead can be reduced further. However, by doing so, there will be a trade-off between the achievable user rate and the reduction in the notification overhead when a subset of antennas is disabled on all sub-channels.

\section{NumERICAL EXAMPLES}

The performance of the proposed beamformer designs using the average number of backlogged bits for various arrival rates is demonstrated. The model considers a cell-edge scenario wherein the path loss between users and BSs is fixed to 0 $\mathrm{dB}$ and the fading channels $\mathbf{h}_{b, k, n}$ are is drawn from i.i.d $\mathcal{C N}(0 ; 1)$. The noise variance and the transmit power are fixed to $N_{0}=30 \mathrm{dBm}$ and $P_{\max }=40 \mathrm{dBm}$, respectively. In all figures, we have denoted the non-coordinated transmission as isolated approach wherein each user is served by single BS, which is assigned during system initialization. Additionally, we also compared with beam-selection scheme, which can be obtained with an additional constraint $\sum_{b \in \mathcal{B}} c_{b, k, n}=1, \forall k \forall n$ in (18), i.e., on any sub-channel, a user is served by one BS only. For unquantized designs, which are used as performance benchmarks, no beamformer overhead is accounted for in the backhaul, i.e., only the user rate is considered in the backhaul overhead. However, for all the quantized designs, beamformers are also included with the users data. The amplitude and the phase of each complex entry are uniformly quantized with 8 bits each to avoid any degradation due to quantization. The solutions with constraints (29) and (31) are denoted by $B$ and $A$, respectively. The convex subproblems obtained in each SCA

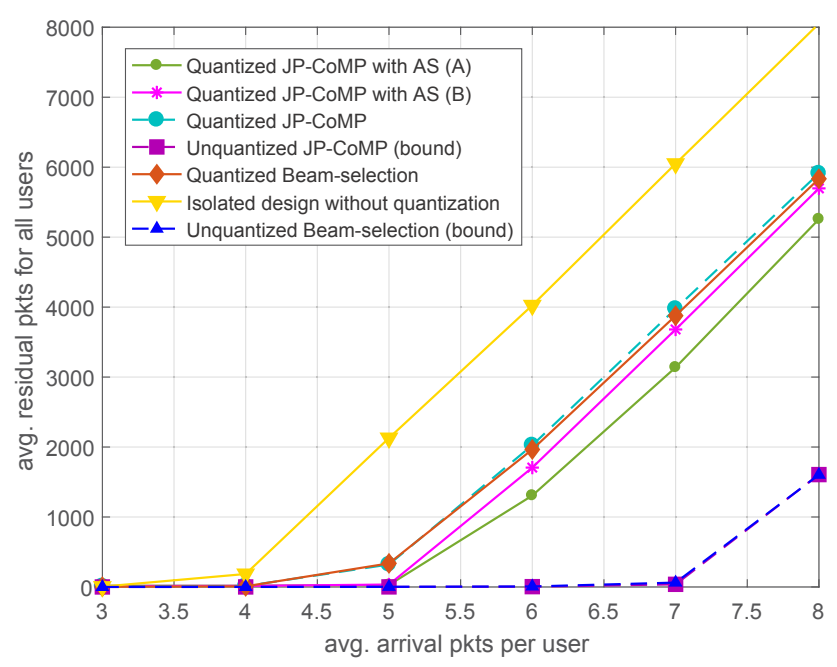

(a). Average number of backlogged packets.

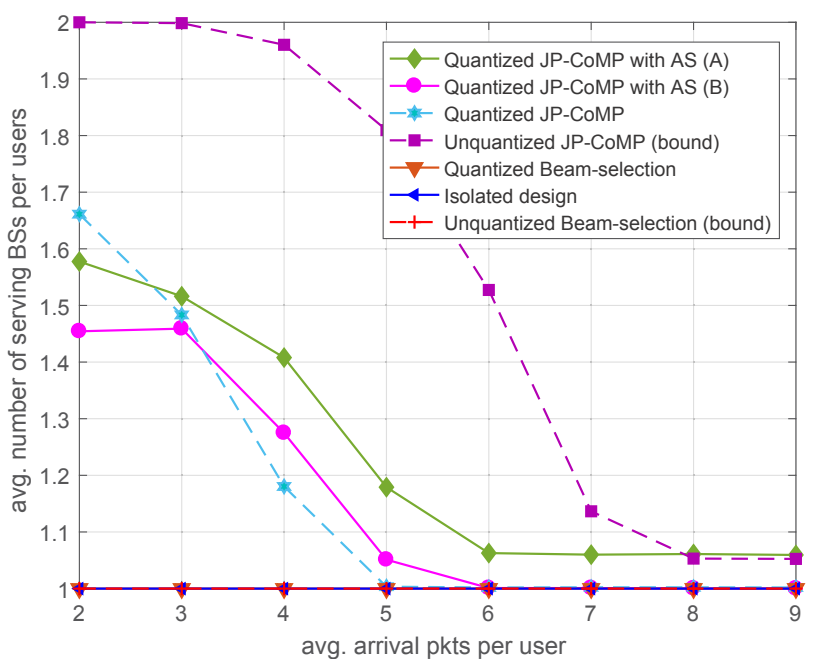

(b). Average number of serving BSs per user.

Fig. 1. Behavior of $\left\{N_{B}, K, N, N_{T}\right\}=\{2,8,2,16\}$ over 500 slots with backhaul capacity 30 and 40 bits, respectively, and $\mu=0.01$. Both phase and amplitude are quantized using 8 bits each to avoid any loss due to quantization.

step are modeled and solved by using [18]. The beamformer to data fraction $\mu=0.01$ is assumed in all simulations, i.e., each beamformer is used for 100 data symbols.

Fig. 1(a) plots the total number of residual bits in the system for all the proposed schemes by varying the average packet arrival rates of the users. Comparing the unquantized designs, the centralized CoMP has the least number of residual packets, followed by the beam selection scheme and then by the isolated scenario. However, in the presence of quantization, the total number of backlogged packets increases significantly for the two cooperative designs wherein the beamformers are designed at the $\mathrm{CC}$. In the presence of beamformer quantization, the proposed antenna selection designs performs noticeably better than the other two quantized schemes by resorting to antenna selection. These gains will be magnified by the number of data symbols present in each coherence block (sub-channel). As the average arrival rate increases per user, 


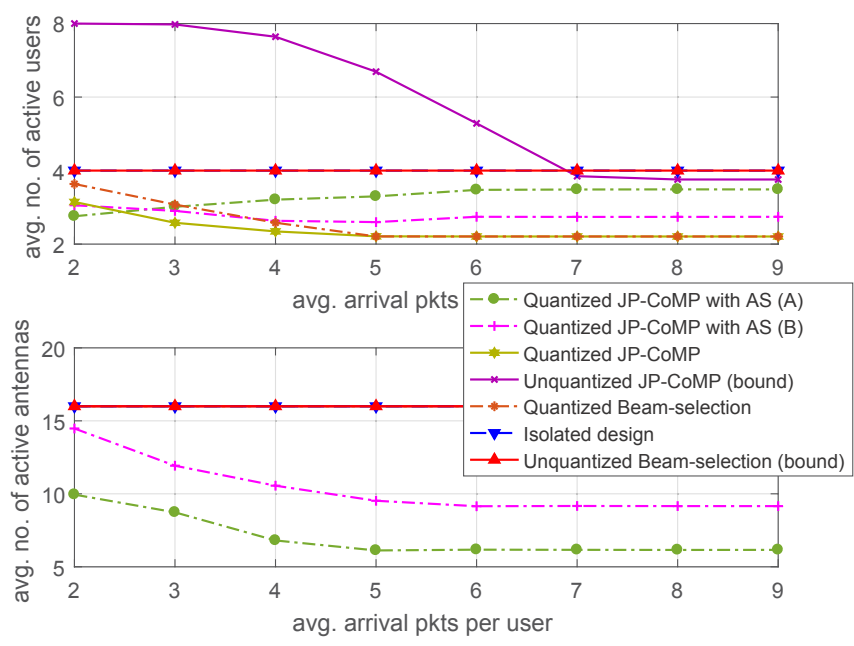

Fig. 2. Average number of active antennas used for active transmissions and the average number of active users per BS for a same system settings.

BS coordination is preferred over cooperation, which can be seen from Fig. 1(b) by a drop in the number of serving BSs per user. It is obtained by taking average of the maximum number of BSs serving each user over all sub-channels.

As discussed in Section IV, Fig. 2 illustrates the emphasis of the constraint (31) or $(A)$ on reducing the number of active antennas than restricting the total users count. On the contrary, (29) or (B) manages the backhaul capacity by restricting the number of active users over the antenna selection counterpart. As the arrival rate increases, the objective can be minimized by serving only a subset of users with better channel quality as there will be enough backlogged packets. Thus, by selecting an efficient subset of antennas for transmission, beamformer overhead can also be minimized. This trend is shown in Fig. 2. Moreover, by choosing less number of antennas, the active users count is also increased as shown in Fig. 2. For a fixed quantization bits and backhaul capacity, the antenna selection designs outperform other schemes if either one of the following is true, namely, the number of transmit antennas used by the BSs increases or when the channel coherency is limited, which leads to few data symbols for each beamformer ( $\mu$ increases). The proposed designs has no use if $\delta_{b, k, n} \geq N_{Q}$, i.e., when the notification overhead is greater than the quantization bits.

\section{Conclusions}

We have proposed two transmit beamformer designs for a multi-carrier joint processing coordinated multi-point cellular systems wherein a centralized controller was used to design, quantize and feed back them to the respective base station (BS) via a finite backhaul link along the associated user data. Due to the restrictions laid by the finite backhaul capacity and the beamformer design, it became a nonconvex combinatorial problem with the objective of minimizing the total number of backlogged packets. We resorted to the successive convex approximation technique to address the nonconvex constraints and linear relaxation for the binary variables. Furthermore, we regularized the objective with an entropy measure of the relaxed variables. The overhead due to fixed beamformer quantization becomes a single dominant factor as the number of transmit antennas increases. Hence, we have proposed two solutions based on antenna selection wherein few insignificant antennas were disabled to provide more room for users data in the backhaul. Numerical results were shown to justify the benefits of the proposed design. Even though antenna selection may not be a wise decision while multiplexing users spatially, it turns out that for our current problem, it is in fact beneficial.

\section{REFERENCES}

[1] D. Gesbert, S. Hanly, H. Huang, S. S. Shitz, O. Simeone, and W. Yu, "Multi-cell mimo cooperative networks: A new look at interference," IEEE J. Sel. Areas Commun., vol. 28, no. 9, pp. 1380-1408, 2010.

[2] O. Simeone, O. Somekh, H. V. Poor, and S. Shamai, "Downlink multicell processing with limited-backhaul capacity," EURASIP Journal on Advances in Signal Processing, vol. 2009, no. 1, pp. 1-10, 2009.

[3] B. Dai and W. Yu, "Sparse beamforming and user-centric clustering for downlink cloud radio access network," IEEE Access, vol. 2, pp. 13261339, 2014.

[4] J. Zhao, T. Q. Quek, and Z. Lei, "Coordinated multipoint transmission with limited backhaul data transfer," IEEE Trans. Wireless Commun., vol. 12, no. 6, pp. 2762-2775, 2013.

[5] S.-H. Park, O. Simeone, O. Sahin, and S. Shamai, "Joint precoding and multivariate backhaul compression for the downlink of cloud radio access networks," IEEE Trans. Signal Process., vol. 61, no. 22, pp. $5646-5658,2013$.

[6] O. Simeone, A. Maeder, M. Peng, O. Sahin, and W. Yu, "Cloud radio access network: Virtualizing wireless access for dense heterogeneous systems," arXiv preprint arXiv:1512.07743, 2015.

[7] M. Chiang, S. Low, A. Calderbank, and J. Doyle, "Layering as Optimization Decomposition: A Mathematical Theory of Network Architectures," Proceedings of the IEEE, vol. 95, no. 1, pp. 255-312, Jan 2007.

[8] K. Seong, R. Narasimhan, and J. Cioffi, "Queue Proportional Scheduling via Geometric Programming in Fading Broadcast Channels," IEEE J. Sel. Areas Commun., vol. 24, no. 8, pp. 1593-1602, 2006.

[9] K. K. Mukkavilli, A. Sabharwal, E. Erkip, and B. Aazhang, "On beamforming with finite rate feedback in multiple-antenna systems," IEEE Trans. Inf. Theory, vol. 49, no. 10, pp. 2562-2579, 2003.

[10] D. J. Love, R. W. Heath Jr, W. Santipach, and M. L. Honig, "What is the value of limited feedback for mimo channels?" IEEE Commun. Mag., vol. 42, no. 10, pp. 54-59, 2004.

[11] P. Xia and G. B. Giannakis, "Design and analysis of transmitbeamforming based on limited-rate feedback," in 60th Vehicular Technology Conference, vol. 3. IEEE, 2004, pp. 1653-1657.

[12] J. C. Roh and B. D. Rao, "Performance analysis of multiple antenna systems with vq-based feedback," in Thirty-Eighth Asilomar Conference on Signals, Systems and Computers, 2004. Conference, vol. 2. IEEE, 2004, pp. 1978-1982

[13] B. Mondal and R. W. H. Jr., "Performance analysis of quantized beamforming mimo systems," IEEE Trans. Signal Process., vol. 54, no. 12 , pp. 4753-4766, Dec 2006.

[14] G. Venkatraman, A. Tölli, M. Juntti, and L.-N. Tran, "Traffic Aware Resource Allocation Schemes for Multi-Cell MIMO-OFDM Systems," IEEE Trans. Signal Process., vol. 64, pp. 2730-2745, Jun. 2016.

[15] G. Scutari, F. Facchinei, and L. Lampariello, "Parallel and Distributed Methods for Constrained Nonconvex Optimization-Part I: Theory," IEEE Trans. Signal Process., no. 99, pp. 1-1, 2016.

[16] E. J. Candes, M. B. Wakin, and S. P. Boyd, "Enhancing Sparsity by Reweighted $\ell_{1}$ Minimization," Journal of Fourier analysis and applications, vol. 14, no. 5-6, pp. 877-905, 2008.

[17] G. Venkatraman, A. Tölli, J. Kaleva, and M. Juntti, "Transmit beamformer and quantization design for multi-carrier CRAN CoMP," in IEEE Global Conference on Signal and Information Processing GlobalSIP. IEEE, Dec 2016.

[18] M. Grant and S. Boyd, "CVX: Matlab software for disciplined convex programming, version 2.0 beta," http://cvxr.com/cvx, Sep. 2013. 